\title{
TERAPIA OCUPACIONAL EN UN HOSPITAL GENERAL DE AGUDOS DE LA CIUDAD AUTÓNOMA DE BUENOS AIRES. ARGENTINA
}

\author{
OCCUPATIONAL THERAPY IN ACUTE CARE AT CIUDAD AUTÓNOMA DE BUENOS \\ AIRES. ARGENTINA
}

\section{Mariela Alejandra Pérez Riffo ${ }^{1}$}

\begin{abstract}
Resumen:
Introducción: El rol de Terapia Ocupacional (T.O.) como lo llevamos a cabo en nuestros hospitales generales de agudos de la CABA(Ciudad Autónoma de Buenos Aires, Argentina), sea por falta de recursos humanos, características institucionales, o causas socioeconómicas, posee características particulares; es por ello que las intervenciones tienen un perfil diferente a los que encontramos en las publicaciones disponibles (Affleck, A., Lieberman, J.P. \& Rohrkeper, K., 1986; Bondac, S., Hermann, V., Frost, L., Lashgari, D., Finnen, L. \& Alexander, H., 2009; Eyres, L. \& Unsworth, C., 2005; Giesbrecht, B., 2006; Raush, G. \& Melvin, J., 1986). Objetivos: a) Estimar la distribución de frecuencias y cantidad de intervenciones de T.O. b) Determinar si el promedio de edad y la presencia de cuidador son diferentes según el tipo de intervenciones de Terapia Ocupacional.

Material y Método: Se incluyeron 141 registros de pacientes adultos internados en un Hospital de Agudos que hayan recibido al menos 1 intervención (junio 2008-junio 2009). Estudio cuantitativo, analítico (comparativo a muestras independientes), prospectivo, longitudinal y observacional de cohorte (no experimental).

Resultados: las intervenciones más prevalentes son la realización de interconsultas para pedir información; el uso de las actividades de la vida diaria; educación en desacondicionamiento; posicionamiento; sugerencia o realización de adaptaciones ambientales, férulas entre otras. Se hallaron diferencias significativas entre la edad y uso de actividades de la vida diaria, educación en uso adecuado de la mecánica corporal, posicionamiento, estimulación del alerta, educación en conservación de energía y protección articular, entre otras.

Así mismo se encontraron diferencias significativas entre la presencia de cuidador y educación en desacondicionamiento por un lado y consultoría en organización de cuidados por el otro.

Conclusiones: el trabajo muestra de acuerdo a la realidad institucional y estadio de los pacientes las intervenciones más prevalentes en un hospital de agudos. Se halló relación significativa entre la edad y las diferentes intervenciones analizadas, siendo que a mayor edad son más frecuentes las intervenciones educativas en relación a uso adecuado de la mecánica corporal, desacondicionamiento. También se encontró relación significativa con el uso de las actividades de la vida diaria, posicionamiento, uso de adaptaciones personales, ambientales y estimulación del alerta.
\end{abstract}

Palabras clave:

Hospital de agudos- terapia ocupacional- intervenciones prevalentes

1 Lic. Mariela Alejandra Pérez Riffo

Terapista Ocupacional de planta Hospital General de Agudos B. Rivadavia

011-1533591614 marielaperezto@gmail.com 


\begin{abstract}
Introduction: The role of the Occupational Therapy (OT) in our acute care hospitals in Argentina has peculiar features, whether due to: lack of human resources, institutional characteristics or socioeconomic causes. Therefore the interventions have a different profile to those found in the available literature. (Affleck, A. , Lieberman, J.P. \& Rohrkeper, K., 1986; Bondac, S. , Hermann, V. , Frost, L., Lashgari, D. , Finnen, L. \& Alexander, H. , 2009; Eyres, L. \& Unsworth, C. , 2005; Giesbrecht, B., 2006; Raush, G. \& Melvin, J., 1986).

Objetives: a) To estimate the frequency distribution and the number of OT interventions. b) To determine if the average age and the presence of a caregiver differ depending on the type of OT interventions.

Material and Methods: 141 records of adult inpatients from the Acute Care Hospital who had at least one intervention were included. Comparative study to independent samples, prospective observational and longitudinal (june 2008-june 2009).

Results: The most prevalent are interconsultations for information, use of daily life activities, education on deconditioning, positioning, suggestion or conducting for environmental adaptations, splints, among others. Major differences were observed between the age and the use of daily life activities, education on the proper use of body mechanics, positioning, stimulation of the awareness, training for energy conservation and joint protection, along with others. Also, significant differences were found between the presence of a caregiver and education on deconditioning and consultancy on care management.

Conclusions: The study shows the most prevalent interventions in an acute care hospital, taking into account the institutional situation and the state of patients. Significant relationship between inpatients' age and the different interventions such as use of daily life activities, education on the proper use of body, positioning, stimulation of the awareness was found.
\end{abstract}

Keywords:

Acute care- Occupational Therapy- predominated interventions

\section{INTRODUCCIÓN}

La práctica de terapia ocupacional (T.O.) en Hospitales Generales de Agudos tiene sus antecedentes en el mundo a partir de la década del 80 (Raush \& Melvin, 1986).

En los hospitales del Gobierno de la Ciudad de Buenos Aires (GCBA) se inicia su inserción sistemática, a partir del inicio de las actividades de la Residencia y Concurrencia de T.O. en 1998. Dicho sistema de Residencia y Concurrencias fue creado por decreto $\mathrm{N}^{\circ} 586$ del 24/7/96 (Boletín Oficial 20335, pág. 105-107).

De la bibliografía publicada (Pub Med 1980 - 2008 "Occupational Therapy and acute care", "occupational therapy and inpatient", "rehabilitation and acute care", "acute care settings and occupational therapy") se encuentran artículos donde se citan las intervenciones que realizan los terapistas ocupacionales con mayor frecuencia en los hospitales generales, no obstante, no se han encontrado trabajos científicos que mencionen cuáles son las que prevalecen. En el mundo la inserción de T.O. en los hospitales se brinda a través de prácticas más especializadas por ejemplo por patologías (lesión medular, accidente cerebro-vascular, traumatismo craneoencefálico, patologías traumatológicas, entre otras (Affleck \& cols, 1986; Bondac \& cols, 2009; Eyres et al, 2005; Giesbrecht, 2006; Griffin, S. \& Mcconnell, D., 2001), y en otros casos por grupo etario. (Raush, \& Melvin, 1986). Es decir el rol de T.O. como lo llevamos a cabo en nuestros hospitales de la CABA, Argentina, sea por falta de recursos humanos, sea por características institucionales, sea por causas socioeconómicas, posee características particulares al que observamos en el resto del mundo. Es por ello que las intervenciones tienen un perfil diferente en algunos aspectos a los que encontramos en las publicaciones disponibles, sin embargo las competencias de la T.O. de involucrar a las personas en situaciones vitales y significativas se comparten en todo el mundo. (Fernández Cerrato, M. y Fernández Huete, J., 2005)

Es también de destacar que tampoco se encuentran antecedentes donde se investigue las intervenciones de T.O. relacionadas con variables donde podrían hacerse más pertinentes algunas intervenciones sobre otras.

Sólo se ha hallado un trabajo cualitativo donde fundamenta el porqué los terapistas centran su atención en las actividades de la vida diaria durante la atención en el cuidado agudo por sobre el resto de las áreas del desempeño ocupacional (Gage, M., Cook Valiant, J. \& Friday-Field, K., 1997).

La intención de este trabajo es mostrar las intervenciones documentadas por los terapistas ocupacionales en un hospital general utilizando la terminología actual del marco de referencia (Fernández Cerrato y otro, 2005) y la categorización realizada en Terapia Ocupacional del Hospital Rivadavia (Pérez, M., Casal, C., Cirone, M., González, A., Parraquini, L., Bracco Croceri, F., Vadell, A. y Oromi, M., 2008) y su prevalencia según ciertas variables: edad y presencia de cuidador. 
Es por ello que en pacientes internados se desea:

1. Estimar la distribución de frecuencias de las intervenciones de T.O. llevadas a cabo en las salas de internación de pacientes adultos.

2. Estimar la distribución de frecuencias número de intervenciones de TO llevadas a cabo en las salas de internación de pacientes adultos.

3. Determinar si el promedio de edad es diferente según el tipo de intervenciones de Terapia Ocupacional.

4. Determinar si los distintos tipos de intervenciones de Terapia Ocupacional son diferentes según presencia de cuidador.

\section{Población}

Criterios de inclusión: documentación que constituye el registro interno de las intervenciones realizadas de pacientes adultos (mayores de 18 años) internados en las salas del hospital general y que hayan recibido al menos 1 intervención de T.O.

Criterios de exclusión: se excluirán aquellas observaciones en las cuales por situaciones relacionadas al sistema de salud no se pudo registrar la intervención de T.O.

Pacientes que no acepten que se registre su intervención para fines de investigación.

Criterios de eliminación: ausencia de registro escrito en fichas de evolución diaria.

Coordenadas témporo-espaciales: salas del Hospital General de Agudos B Rivadavia durante el período junio 2008 - junio 2009.

Cada paso de la investigación fue llevado a cabo por la autora del artículo: elaboración del protocolo, registro de datos (a través de la lectura de la documentación interna de las intervenciones realizadas por terapia ocupacional), análisis de los resultados y la elaboración de esta publicación.

Tipo de estudio: cuantitativo, analítico (comparativo a muestras independientes), prospectivo, longitudinal y observacional de cohorte (no experimental).

Variables en estudio: Para la operacionalización de las variables se utilizó el Marco para la Práctica de TO (Fernández Cerrato y otro, 2005) y la categorización elaborada por las terapistas del hospital (Pérez y otros, 2008).

Es por ello que se entiende por Intervenciones de T.O. al: proceso por el cual el terapista ocupacional actúa para cambiar el entorno, demandas de la actividad, características del paciente, habilidades y patrones del desempeño con el fin de mejorar el desempeño en las ocupaciones del paciente durante su estadía hospitalaria (Fernández Cerrato y otro, 2005). Descripción en el registro interno de cada una de las siguientes categorías que se detallarán a continuación.

Dentro de las posibles intervenciones que puede realizar un terapista ocupacional durante la estadía hospitalaria se encuentran (Fernández Cerrato y otro, 2005):

\subsection{Las entrevistas/evaluaciones}

1.2 La implementación del uso terapéutico del yo (Fernández Cerrato y otro, 2005): es la planificación del uso de la personalidad, introspección, percepción y juicio como parte del proceso terapéutico. Se incluyen en esta categoría uso de escucha activa, vehiculización de necesidades, acompañamiento, confort, confrontación, validación de emociones, refuerzo positivo, uso del silencio. (Pérez y otros, 2008).

1.3 El uso terapéutico de las ocupaciones y actividades que incluye:

A) Ocupaciones-actividades básicas: son las actividades donde el paciente se involucra dentro de su entorno real (Fernández Cerrato y otro, 2005; Pérez y otros, 2008)

B) Actividades con propósito: son las que permiten al paciente entrenar conductas o actividades encaminadas a conseguir los objetivos dentro de un entorno terapéutico (Fernández Cerrato y otro, 2005).

b.1. práctica simulada de AVD, o AIVD dentro de la sala, por ejemplo practicar la actividad de alimentación cortando masa con cuchillo/tenedor.

C) Métodos preparatorios: preparan al paciente para el desempeño de una ocupación (Fernández Cerrato y otro, 2005)

\section{c.1: Confección y/o sugerencia de adaptaciones:}

personales: realización de cambios en los objetos y/o tareas para facilitar el desempeño: engrosadores, cuchillo con toma modificada, plato con clavos, antideslizantes, mangos alargados, salea. (Fasoli, L., 2008; Maher, C. \& Bear-Lehman, J., 2008; Pérez y otros, 2008). 
ambientales: realización de cambios en el espacio físico o el ambiente para facilitar un óptimo desempeño del paciente y de las interacciones con sus cuidadores: altura del mobiliario, ubicación del mobiliario y elementos de uso frecuente, luz, ventilación, accesos.James, AB., 2008; Maher et al, 2008; Pérez y otros, 2008).

c.2: Confección de férulas y dispositivos externos para evitar contracturas-deformidades, brindar confort, disminuir inflamación, dolor, estabilizar/fijar un segmento: valvas de reposo, estabilizadoras de muñeca, valvas para codo, cuñas, almohadas, rollos, aros, tabla para evitar equino, apoya pie en L. (Pérez y otros, 2008). c.3: Actividades ergométricas (Flimn, N., Jackson, J., Gray, J. \& Zemke, R., 2008; Pérez y otros, 2008).

c.4 Estímulos para facilitar atención, orientación, memoria, esquema corporal, percepción, praxias. (Quintana, LA., 2008; Radomski, MV. \& Schols Davis, E., 2008).

c.5 Posicionamiento en decúbito lateral, supino, prono, sentado, ascenso en la cama, alineación de segmentos corporales (James, AB., 2008).

1.4. Asesoría/consultoría (dirigidas al paciente y/o familia-cuidador): espacio terapéutico en el cual en conjunto con el paciente frecuentemente mediante la técnica de resolución de problemas se estimula la expresión de conocimientos previos, creencias, mitos, costumbres, se refuerza positivamente lo conocido, utilizado y beneficioso, se explicita efectos negativos de lo erróneo y trascendente, se brinda un espacio para clarificar información con los profesionales u otra fuente de conocimiento, promueve la identificación de conductas factibles para llevar a cabo, se facilita material escrito pre-formado, se facilita la práctica real cuando es posible y se abordan los siguientes aspectos (Pérez y otros, 2008).

- Principios de conservación de energía (se deberá especificar área trabajada) (Fasoli, S., 2008; Lynn, Y., 2008).

- Principios de protección articular (se deberá especificar principio trabajado) (Fasoli, S., 2008; Lynn, Y., 2008).

- Manejo de síntomas: dolor, stress, ansiedad, miedo, angustia, insomnio, disnea, fatiga en relación a su desempeño diario (Fasoli, S., 2008; Lynn, Y., 2008; López Rosetti, D., 2000).
- Preparación para el alta: incluye considerar las modificaciones ambientales necesarias en el domicilio, los cambios de rutinas, conductas requeridos, los problemas identificados por el paciente/cuidador ante este acontecimiento (Pérez y otros, 2008).

- Organización de rutinas diarias, mantenimiento de la salud (Fernández Cerrato y otro, 2005; Pérez y otros, 2008).

- Organización de cuidados (Fernández Cerrato y otro, 2005; Pérez y otros, 2008).

- Manejo de situaciones problema no especificadas arriba.

1.5. Educación: (dirigidas al paciente y/o familia-cuidador). Se relaciona con el asesoramiento que realiza el profesional en relación a los temas citados en asesoría y otros.

- Uso adecuado de la mecánica corporal: postura para movilizar al paciente y manera de usar los elementos necesarios para tal fin (saleas, arnés, entre otros), forma de incorporarse, trasladar objetos pesados ( $\mathrm{Fa}$ soli; S., 2008; Lynn, Y., 2008; Maher \& col, 2008).

- Medidas de bioseguridad en sala, cuidado propio, de otros, transmisión de infecciones

- En equipamiento/ tecnologías: respirador, vía periférica, central, cánula nasal, máscara de oxígeno, sonda nasogástrica, gastrostomía, sondas de débito, sonda vesical, uroset, colostomía, drenajes, entre otros. La educación se imparte en relación a su participación en actividades, grado de interferencia o no en las mismas. (Affleck \& cols, 1986; Pérez, M., 2006).

- Principios de protección articular (Lynn, Y., 2008).

- Principios de conservación de energía (Lynn, 2008; Maher \& col, 2008).

- Manejo de síntomas: dolor, inflamación, disnea, fatiga (Fasoli, 2008; Lanier Pierce, S., 2008; López Rosetti, 2000; Lynn, 2008; Maher \& col, 2008).

- Prevención del desacondicionamiento: úlceras por presión, edema, contracturas, deformidades, hipotensión ortostática, trombosis venosa profunda, dis- 
minución de fuerza, amplitud articular, resistencia (Gill, T., Allore, H. \& Guo, Z., 2004; Gillis, A., MacDonald, B. \& Macksaac, 2008; Pérez, 2006).

1.6. Interconsultas (Pérez y otros, 2008; Pérez, 2006).

- Interconsulta para pedir o recibir información: comunicación con uno, alguno o todos los miembros del equipo de profesionales que asisten al paciente a fin de solicitar o recibir datos sobre su estado clínico actual, diagnóstico, pronóstico, datos histórico-familiares, demográficos, sociales, etc.

- Interconsulta para transmitir información: comunicación con uno, alguno o todos los miembros del equipo de profesionales que asisten al paciente a fin de brindar datos sobre situaciones problemas del paciente-familia detectadas en la sesión de terapia ocupacional o verbalizadas por la familia.

- Interconsulta para vehiculizar la participación de otro profesional: sugerencia (escrita o verbal) por parte del terapista de la inclusión de otro profesional en la asistencia del paciente-familia según la situación emergente que se haya detectado.

- Interconsulta para vehiculizar la necesidad de un tercer nivel: comunicación con algunos o todos los miembros del equipo de profesionales que asisten al paciente a fin de sugerir la gestión de un tercer nivel (seguimiento consultorios externos; rehabilitación, geriatría, psiquiatría, otros).

- Interconsulta para prolongar tratamiento: comunicación con uno, alguno o todos los miembros del equipo de profesionales que asisten al paciente a fin de plantear la necesidad de que el paciente permanezca internado.

1. Cantidad de intervenciones de TO (número de intervenciones.)

2. Edad medida en años según consta en historia clínica.

3. Estadio de la enfermedad medida en escala nominal: final de vida o no según referencia del médico tratante.

4. Presencia de familia/cuidador medida en escala nominal (presente - ausente) según referencia del paciente $y / o$ equipo de profesionales.

\section{Variable a controlar}

Estado clínico del paciente durante su internación:

Se considera paciente en estado clínico agudo a quien tiene una enfermedad con un inicio y un fin claramente definidos. Generalmente son de corta duración, aunque no hay un consenso en cuanto a qué plazos definen a una enfermedad como aguda y cuáles como crónica. El término agudo no dice nada acerca de la gravedad de la enfermedad (Beneit Medina, P., 1994; Piedrola, G., 2001).

Se considera paciente en estado clínico agudo cronificado a aquel que permanece en la institución hospitalaria con un proceso incurable, cuya etiología múltiple tiene un desarrollo poco predecible, y que por diferentes causas: familiares (sin familia o familia no continente), sociales (falta de lugar de $3^{\circ}$ nivel para su derivación) continúa en la institución sin criterio para su internación en el hospital general de agudos. (Beneit Medina, P., 1994; Piedrola, G., 2001).

\section{Muestra y Muestreo}

Para una prevalencia mínima esperada de intervenciones de TO del 10\% (mínimo 5\%, máximo 15\%) con un error alfa $=0.05$ el tamaño muestral necesario es de 140 casos. El procedimiento de muestreo será aleatorio simple.

\section{Análisis Estadístico}

Los datos se analizaron en base de datos Excel, se empleó el paquete estadístico Medcalc 9.5 y VCCStat 2.0. Para las variables se estableció la distribución de frecuencias y/o los porcentajes en relación con el total de casos. Para aquellas medidas en escala ordinal o superior se computarón las siguientes estadísticas: número de casos, valor mínimo y máximo hallado, media aritmética, desvío estándar.

Se realizaron las siguientes pruebas de significación Chi cuadrado, análisis de la variancia, Kruskall-Wallis, e intervalos de confianza, siendo el nivel de significación alfa $=0.05$.

\section{Reparos Éticos}

El protocolo fue aprobado por el Comité de ética y el Departamento de Docencia e Investigación del Hospital de Agudos B. Rivadavia. 
Se mantuvo la confidencialidad de los datos personales de los registros de seguimiento de Terapia Ocupacional de los pacientes que cumplieron con los criterios mencionados en apartado población.

Los pacientes que se negaron a que se registre la intervención no fueron incluidos en la investigación.

\section{Descripción de la muestra}

La muestra estuvo constituida por 141 registros internos de pacientes, el $58,2 \%$ femenino y el resto masculino.

El promedio de edad fue $67,1 \pm 18,5$ años (mínimo= 18, mediana 70, máximo=94)

El promedio de días de internación fue de 27,08 días $\pm 37 ;($ mínimo=2, mediana 18, máximo=369).

El promedio de sesiones recibidas por los pacientes fue de $7 \pm 6$; (mínimo=1, mediana 5, máximo $=30$ sesiones por paciente).

El 73,8 \% tenían cuidador.

El 18,4\% se encontraban en etapa final de vida según historia clínica.

\section{Resultados}

Objetivo $N^{\circ}$ 1: Estimar la distribución de frecuencias de las intervenciones de T.O. Ilevadas a cabo en las salas de internación de pacientes adultos:

A partir del análisis de los resultados se encuentran las siguientes intervenciones de TO que se realizan con mayor frecuencia. Dichos datos se muestran en las siguientes tablas (1 a 6 ).

Tabla $N^{\circ} 1$

Distribución de frecuencia de la Intervención Interconsulta

\begin{tabular}{|c|c|c|c|}
\hline Intervención Interconsulta & $\mathrm{N}$ & $\%$ & IC95\%* \\
\hline Pedir información & 133 & 94,3 & $89,1-97,5$ \\
\hline Transmitir información & 68 & 48,2 & $39,8-56,8$ \\
\hline $\begin{array}{c}\text { Vehiculizar intervención de otro } \\
\text { profesional }\end{array}$ & 27 & 19,1 & $13,2-26,8$ \\
\hline
\end{tabular}

*intervalo de confianza del $95 \%$

Se puede observar que dentro de esta categoría la interconsulta para pedir información (es decir la acción de indagar con los profesionales médicos sobre el estado clínico del paciente y las contraindicaciones para involucrarse en el tratamiento de TO) es la más prevalente.

Tabla No 2

Distribución de frecuencias correspondiente a la Intervención Uso terapéutico de las actividades

\begin{tabular}{|c|c|c|c|}
\hline $\begin{array}{c}\text { Intervención Uso terapéutico de las } \\
\text { actividades }\end{array}$ & $\mathrm{N}$ & $\%$ & IC 95\% \\
\hline Actividades de la vida diaria & 96 & 68,1 & $59,6-75,5$ \\
\hline Actividad significativa & 16 & 11,3 & $6,8-18,1$ \\
\hline Actividad de tiempo libre & 7 & 5,0 & $2-10,0$ \\
\hline Actividad propositiva & 4 & 2,8 & $0,8-7,1$ \\
\hline Actividades de colaboración & 1 & 0,7 & $0-3,9$ \\
\hline
\end{tabular}

Se releva de esta tabla que las actividades de la vida diaria son las intervenciones más frecuentes de esta categoría.

Tabla $\mathrm{N}^{\circ} 3$

Distribución de frecuencias de la Intervención Educación

\begin{tabular}{|c|c|c|c|}
\hline Intervención educación & $\mathrm{N}$ & $\%$ & $\mathrm{IC} 95 \%$ \\
\hline Desacondicionamiento & 90 & 63,8 & $55,3-71,6$ \\
\hline Manejo síntomas & 32 & 22,7 & $16,3-30,7$ \\
\hline Conservación de energía & 27 & 19,1 & $13,2-26,8$ \\
\hline Equipamiento soporte & 23 & 16,3 & $10,8-23,7$ \\
\hline Mecánica corporal & 13 & 9,2 & $5,2-15,6$ \\
\hline Protección articular & 3 & 2,1 & $0,4-6,1$ \\
\hline Bioseguridad & 2 & 1,4 & $0,2-5$ \\
\hline
\end{tabular}

Se destaca la prevalencia de la educación en desacondicionamiento, es decir la información que se facilita a paciente y cuidador acerca de los efectos del reposo en cama que deviene de la estadía hospitalaria.

Tabla $\mathrm{N}^{\circ} 4$

Distribución de frecuencias de la Intervención correspondiente a Métodos preparatorios

\begin{tabular}{|c|c|c|c|}
\hline Intervención Método preparatorio & $\mathrm{N}$ & $\%$ & $\mathrm{IC} 95 \%$ \\
\hline Posicionamiento & 73 & 51,8 & $43,2-60,2$ \\
\hline Adaptación ambiental & 68 & 48,6 & $39,8-56,8$ \\
\hline Férulas & 47 & 33,3 & $25,8-41,8$ \\
\hline Adaptación personal & 44 & 31,2 & $23,8-39,6$ \\
\hline Actividades ergométricas & 23 & 16,3 & $10,8-23,7$ \\
\hline Estimulación del Alerta & 18 & 12,8 & $7,9-19,7$ \\
\hline
\end{tabular}

El posicionamiento (cambiar de posición en la cama, sentarlo al borde de la misma, alinear segmentos corporales) constituye la intervención que se realiza con mayor frecuencia dentro de esta 
categoría. La realización de adaptaciones ambientales: cambiar alturas, acercar elementos, modificar el ambiente para facilitar el desempeño también se observa con frecuencia.

\section{Tabla $N^{\circ} 5$}

Distribución de frecuencias de la Intervención Uso terapéutico del Yo

\begin{tabular}{|c|c|c|c|}
\hline Intervención Uso terapéutico del yo & $\mathrm{N}$ & $\%$ & IC 95\% \\
\hline Escucha activa & 66 & 46,8 & $38,4-55,4$ \\
\hline Vehiculización necesidades & 56 & 39,7 & $31,7-48,3$ \\
\hline Acompañamiento & 35 & 24,8 & $18,1-32,9$ \\
\hline Confort & 32 & 22,7 & $16,3-30,7$ \\
\hline Confrontación & 29 & 20,6 & $14,4-28,4$ \\
\hline Refuerzo positivo & 27 & 19,1 & $13,2-26,8$ \\
\hline Silencio & 21 & 14,9 & $9,7-22,1$ \\
\hline Validación & 10 & 7,1 & $3,6-13$ \\
\hline
\end{tabular}

En esta categoría se destacan fundamentalmente dos intervenciones: la escucha activa y la vehiculización de necesidades.

Tabla $N^{\circ} 6$

Distribución de frecuencias de la variable Consultoría

\begin{tabular}{|c|c|c|c|}
\hline Intervención Consultoría & $\mathrm{N}$ & $\%$ & IC 95\% \\
\hline Preparación al alta & 36 & 25,5 & $18,7-33,7$ \\
\hline Organización rutinas & 21 & 14,9 & $9,7-22,1$ \\
\hline Organización cuidados & 16 & 11,3 & $6,8-18,1$ \\
\hline Resolución problemas & 13 & 9,4 & $5,2-15,6$ \\
\hline Manejo de síntomas & 1 & 0,7 & $0-3,9$ \\
\hline Protección articular & 0 & 0 & 0 \\
\hline Conservación de energía & 0 & 0 & 0 \\
\hline
\end{tabular}

Se puede observar que la prevalencia, en general, de estas intervenciones, es baja en nuestra muestra, siendo la más frecuente las intervenciones relacionadas a la preparación para el alta. Dentro de esta categoría se incluyen la identificación de áreas problemas en el hogar y la búsqueda de soluciones de manera conjunta para los problemas detectados en su hogar.

\section{Objetivo $\mathrm{N}^{\circ}$ 2: Estimar la distribución de frecuen- cias de la cantidad de intervenciones de TO lleva- das a cabo en las salas de internación de pacien- tes adultos}

Las tablas subsiguientes $\left(\mathrm{N}^{\circ} 7,8\right.$ y 9$)$ dan respuesta al mencionado objetivo. En las mismas se detalla la distribución de frecuencia de la cantidad de veces que se realizaron las intervenciones de Terapia Ocupacional.
Tabla $\mathrm{N}^{\circ} 7$

Distribución de frecuencia de la cantidad de veces que se lleva a cabo la intervención de las actividades de la vida diaria

\begin{tabular}{|c|c|c|c|}
\hline $\begin{array}{c}\text { Cantidad de veces que se } \\
\text { realizó AVD }\end{array}$ & $\mathrm{N}$ & $\%$ & IC 95\% \\
\hline 1 & 28 & 29,2 & $20.6-39.5$ \\
\hline 2 & 19 & 19,8 & $12.6-29.4$ \\
\hline 3 & 12 & 12,5 & $6.9-21.2$ \\
\hline 4 & 9 & 9,4 & $4.4-17-1$ \\
\hline Más de 5 & 28 & 29,2 & $20.6-39.5$ \\
\hline Total & 96 & $100 \%$ & \\
\hline
\end{tabular}

Cabe mencionar que esto quiere decir que en el seguimiento de un paciente, según el registro escrito esta intervención se realiza una vez en el $29 \%$ de los casos pero también en el mismo porcentaje de casos más de 5 veces.

Tabla No 8

Distribución de frecuencia de la cantidad de veces que se llevó a cabo la intervención posicionamiento

\begin{tabular}{|c|c|c|c|}
\hline $\begin{array}{c}\text { Cantidad de veces que se } \\
\text { realizó posicionamiento }\end{array}$ & $\mathrm{N}^{\circ}$ & $\%$ & IC $95 \%$ \\
\hline 1 & 18 & $24,6 \%$ & $15.6-36.4$ \\
\hline 2 & 19 & $26 \%$ & $16.8-37.8$ \\
\hline 3 & 9 & $12,3 \%$ & $5.8-22.1$ \\
\hline 4 & 6 & $8,2 \%$ & $3.1-17$ \\
\hline Más de 5 & 21 & $28,8 \%$ & $19.1-40.7$ \\
\hline Total & 73 & $100 \%$ & \\
\hline
\end{tabular}

Se puede observar que esta intervención se realiza con mucha frecuencia por paciente (más de cinco veces en el $28,8 \%$ ).

Tabla $N^{\circ} 9$

Distribución de frecuencia de la cantidad de veces que se realizan interconsultas para pedir información

\begin{tabular}{|c|c|c|c|}
\hline $\begin{array}{c}\text { Cantidad de veces que se rea- } \\
\text { lizó IC p/ pedir información }\end{array}$ & $\mathrm{N}^{\circ}$ & $\%$ & IC 95\% \\
\hline 0 & 8 & $5,70 \%$ & $2.5-10.9$ \\
\hline 1 & 31 & $22,00 \%$ & $15.6-29.9$ \\
\hline 2 & 32 & $22,70 \%$ & $16.3-30.7$ \\
\hline 3 & 22 & $15,60 \%$ & $10.2-22.9$ \\
\hline 4 & 11 & $7,80 \%$ & $4.2-13.9$ \\
\hline Más de 5 & 37 & 26.2 & $19.4-34.4$ \\
\hline Total & 141 & $100 \%$ & \\
\hline
\end{tabular}

En esta categoría la distribución es variable en cuanto a la cantidad de veces que se realizan por paciente las interconsultas. 


\section{Objetivo $N^{\circ} 3$ Relación del promedio de edad y las intervenciones de Terapia Ocupacional}

Se presentan los siguientes resultados:

El promedio de edad entre los pacientes con intervención uso de AVD $(n=96)$ fue $71 \pm 16$ años mientras que entre los que no recibieron dicha intervención $(n=$ 45) fue $58 \pm 20$ años. Las diferencias entre los promedios de edad hallados fueron estadísticamente significativas $(\mathrm{t}=4.21 ; \mathrm{p}<0.0001)$.

Con respecto al promedio de edad entre los pacientes que recibieron la intervención correspondiente a método preparatorio en su sub categoría adaptaciones ambientales $(n=68)$ fue $73 \pm 15$ años del grupo que tuvo la intervención $(n=73)$, mientras que los que no recibieron dicha prestación fue de $61 \pm 20$ años. Las diferencias entre los promedios de edad halladas fueron estadísticamente significativos ( $t=4.12 ; \mathrm{p}<0.0001)$.

Siguiendo con este grupo de intervenciones también se hallaron diferencias significativas entre los prome- dios de edad de los que recibieron la prescripción de adaptaciones personales $(n=44) 73 \pm 14$ años y los que no les prescribieron dichos elementos $(n=97) 64 \pm 20$ años $(\mathrm{t}=2.91 \mathrm{p}<0.0042)$.

Se observa que el promedio de edad entre los pacientes que tuvieron la intervención posicionamiento $(n=73)$ fue $74 \pm 15$ años mientras que, en los que no tuvieron dicha intervención $(n=68)$ fue $59 \pm 19$ años. Las diferencias entre los promedios de edad halladas fueron estadísticamente significativos $(\mathrm{t}=5.11 \mathrm{p}<0.0001)$.

Dentro de la última sub categoría de estas intervenciones de método preparatorio se encuentran diferencias entre los promedios de edad en el grupo que recibió la estimulación en el alerta $(n=18) 78 \pm 13$ años y los que no la recibieron $(n=123) 66 \pm 19$ años. Las diferencias entre los promedios de edad halladas fueron estadísticamente significativas $(\mathrm{t}=2.61 \mathrm{p}<0.009)$.

A continuación se presenta en la tabla $\mathrm{N}^{\circ} 10$ las sub categorías de la Intervención Educación donde se hallaron diferencias significativas entre los promedios de edades:

Tabla $N^{\circ} 10$

Intervención Educación y su relación con el promedio de edad

\begin{tabular}{|c|c|c|c|c|c|c|c|}
\hline \multirow{2}{*}{\begin{tabular}{c} 
Variable \\
\cline { 2 - 7 }
\end{tabular}} & \multicolumn{3}{|c|}{ Sí recibió } & \multicolumn{3}{c|}{ No recibió } & \multirow{2}{*}{ Test de Student } \\
\hline $\begin{array}{c}\text { Educación en el uso de la mecánica cor- } \\
\text { poral }\end{array}$ & 81 & 9 & 13 & 66 & 19 & 128 & \multirow{2}{*}{$\mathrm{t}=4.85 ; \mathrm{p}<0.0001$} \\
\hline $\begin{array}{c}\text { Educación en protección articular } \\
\text { Educación en conservación de energía }\end{array}$ & 45 & 20 & 3 & 68 & 18 & 138 & $\mathrm{t}=-2.15 \mathrm{p}<0.0332$ \\
\hline Educación en desacondicionamiento & 72 & 16 & 90 & 59 & 20 & 51 & $\mathrm{t}=3.70 \mathrm{p}<0.0004$ \\
\hline
\end{tabular}

*Desvío estándar.

\section{ObjetivoN4: Relación de la presencia de cuidador y las intervenciones de Terapia Ocupacional}

En las tablas 11 y 12 se presentan las intervenciones que tuvieron diferencias significativas en relación a la presencia de cuidador dando respuesta al objetivo $\mathrm{N}^{\circ} 4$.
Tabla N ${ }^{\circ} 11$

Relación entre presencia de cuidador y la intervención en educación en desacondicionamiento

\begin{tabular}{|c|c|c|c|}
\hline \multirow{2}{*}{$\begin{array}{c}\text { Presencia } \\
\text { cuidador }\end{array}$} & \multicolumn{2}{|c|}{ Educación desacondicionamiento } & \multirow{2}{*}{ Total } \\
\cline { 2 - 3 } sí & 75 & No & \multirow{2}{*}{104} \\
\cline { 2 - 3 } & $72 \%$ & $28 \%$ & \multirow{2}{*}{37} \\
\hline \multirow{2}{*}{ no } & 15 & 22 & \multirow{2}{*}{141} \\
\cline { 2 - 3 } & $41 \%$ & $59 \%$ & \\
\hline \multirow{2}{*}{ Total } & 90 & 51 & \\
\hline
\end{tabular}

Chi2=10.4; $p=0.0012$. 
Tabla $N^{\circ} 12$

Relación entre presencia de cuidador y la intervención de consultoría en organización de cuidados

\begin{tabular}{|c|c|c|c|}
\hline \multirow{2}{*}{$\begin{array}{c}\text { Presencia } \\
\text { cuidador }\end{array}$} & \multicolumn{2}{|c|}{ Consultoría organización de cuidados } & \multirow{2}{*}{ Total } \\
\cline { 2 - 3 } & sí & No & \\
\cline { 2 - 3 } & 16 & 88 & \multirow{2}{*}{304} \\
\hline \multirow{2}{*}{ no } & $15 \%$ & $85 \%$ & \\
\cline { 2 - 3 } & 0 & 37 & \multirow{2}{*}{141} \\
\hline \multirow{2}{*}{ Total } & 16 & $100 \%$ & \\
\hline
\end{tabular}

Chi2 $=4.9 ; p=0,0256$.

\section{CONCLUSIONES}

- En la población de pacientes internados en un hospital general de agudos de la CABA, Argentina, atendidos por los profesionales de terapia ocupacional podemos esperar que de mínimo:

- $\quad$ El 89,1\% realice interconsultas para pedir información.

- $\quad 59,1 \%$ utilice las actividades de la vida diaria

- 55,3 eduque en desacondicionamiento

- $\quad 43,2 \%$ realice posicionamiento

- $\quad 39,8 \%$ sugiera o realice adaptaciones ambientales

- $\quad 25,8 \%$ diseñe, entrene, e indique férulas

- $38,4 \%$ realice escucha activa

- $\quad 31,7 \%$ vehiculización de necesidades

- En la población de pacientes se realizó con mayor prevalencia 5 o más veces las intervenciones de Actividades de la Vida Diaria, posicionamiento e interconsultas para pedir información.

- Se halló relación significativa entre la edad y las diferentes intervenciones analizadas, siendo que a mayor edad son más frecuentes las intervenciones educativas en relación a desacondicionamiento, uso adecuado de la mecánica corporal. Así mismo se halló relación con el uso de actividades de la vida diaria, posicionamiento, adaptaciones personales y ambientales, así como estimulación del alerta.

- Se halló relación significativa entre la presencia de cuidador con la educación en el desacondicionamiento y con la organización de cuidados.

\section{DisCUSIÓN}

\section{Fortalezas y Debilidades del estudio}

El presente trabajo constituye el eslabón inicial en investigación de la práctica de Terapia Ocupacional en Hospitales Generales de Agudos en Argentina. De la búsqueda bibliográfica para sustentar este trabajo no se han encontrado investigaciones llevadas a cabo por TO que describan las intervenciones que caracterizan el ejercicio profesional, sólo se encuentran publicaciones que mencionan población blanco, descripción del rol pero sin sustento científico (Bondac \& cols, 2009; Do Prado De Carlo, M., Camargo Bartalotti, C. y Muñoz Palm, RdC A., 2004; Gage \& cols, 1997; Raush \& Melvin, 1986), o en otros casos, donde citan al T.O. en equipos o unidades específicas. (Affleck \& cols, 1986; Eyres \& Unsworth, 2005; Giesbrecht, B. , 2006; Griffin \& Macconnell, 2001; Ocello, M. y Rolfi, F., 2006; Sutton, A., 1998)

Una de las debilidades de este estudio se centra en que se realizó una revisión de las intervenciones de los pacientes provenientes de los registros escritos que constituyen la documentación interna de TO. Por lo antedicho existe la posibilidad de subregistro de intervenciones (es decir no escribir todo lo que se realiza en la sesión terapéutica) por parte del equipo tratante de terapistas ocupacionales.

Otra limitación de este estudio podría radicar en la revisión de literatura donde se utilizó la base de datos pub med para la misma como única fuente.

\section{Consideraciones en relación con los resultados:}

En relación a los resultados, me parece oportuno resaltar las siguientes consideraciones que se reflejan en la práctica asistencial del hospital donde me inserto en 
CABA, Argentina, y que podrían explicar las causas de la menor frecuencia en algunas intervenciones.

\section{Intervención Interconsulta}

El hecho relevado que la realización de Interconsultas para pedir información sea la intervención más prevalente de todas, resalta la necesidad diaria de las TO tratantes de conocer el estado del paciente, y de las contraindicaciones para involucrarse en alguna actividad dada la naturaleza aguda de su cuadro clínico. A diferencia de un tercer nivel de rehabilitación en Argentina donde los objetivos y estrategias tienen cierta estabilidad y la comunicación con el equipo está instituida a una vez por semana en algunas ocasiones, o cada 15 días.

No obstante, se podría adjudicar a una de las razones por las cuales no se registre en un $100 \%$ de las intervenciones (en 1 de cada 10 pacientes); esta intervención se relacionaría con que uno de los recursos humanos destinados a la atención de los pacientes de clínica médica se encuentra en formación (perteneciente al Sistema de Residencias y Concurrencias del GCBA, Residente de $1^{\circ}$ año) y acorde a las coordenadas temporales de este estudio se incluyen meses donde el mencionado recurso inicia su inserción en el área de atención de pacientes internados, y donde las destrezas comunicacionales podrían estar en desarrollo y por lo mismo limitar la consulta con los profesionales médicos.

\section{Uso terapéutico de las Ocupaciones}

Las áreas del desempeño y específicamente las actividades de la vida diaria constituyen uno de los ejes de la terapia ocupacional, dado que el objetivo central es obtener la independencia y/ autonomía en las mismas (Fernández Cerrato y otro, 2005; Gage \& cols , 1997; James, 2008). En la práctica de TO en agudos este postulado no es la excepción, es por ello que prevalece la intervención en un 68\% IC 59.6-75.5.

En este caso la razón por la cual no se realiza en el $100 \%$ de la población se relaciona con que los pacientes se encuentran atravesando una internación por una patología "aguda" con lo cual el estado clínico (síntomas fluctuantes, síntomas exacerbados ej. dolor), el estadio de la enfermedad (etapa final de vida o no), las contraindicaciones por la enfermedad o el negativismo a partici- par, son algunas de las razones por las cuales no se lleva a cabo en el $100 \%$ de los casos dicha intervención.

Si bien el uso de actividades significativas tiene una baja prevalencia en esta muestra, se explicaría porque son intervenciones que con mayor frecuencia se realizan con pacientes en etapa final de vida; en este estudio, dicho grupo, está conformado por el $18 \%$ de la muestra.

Las intervenciones menos prevalentes dentro del uso terapéutico de la actividad (actividades de tiempo libre, colaboración) coinciden con lo mencionado por pacientes en otros estudios (Gage \& cols, 1997) sobre lo que les preocupa/interesa a los pacientes durante una hospitalización aguda. Es decir priorizan el alivio de síntomas, la escucha, comunicación, abordaje integral, participación en su cuidado y no las actividades de tiempo libre.

Observamos en la práctica clínica en los hospitales de la CABA, que, cuando se llevan a cabo dichas actividades de colaboración/tiempo libre, se relaciona con pacientes que se encuentran con internaciones prolongadas a la espera de un tercer nivel (geriátrico-centro de rehabilitación) o carentes de lugar para el alta y que se han "cronificado" en la institución.

\section{Intervención Educación}

La prevalencia de esta intervención, educación en desacondicionamiento, esto significa explicarle a ambos usuario-paciente y cuidador-los efectos del reposo en cama (Gill \& cols, 2004; Gillis \& cols, 2008; Pérez, 2006), tal como la aparición de úlceras por presión, debilidad generalizada a predominio de los músculos antigravitacionales, hipotensión postural, atrofia por desuso, desorientación témporo-espacial, entre otras, se implementa en el 63,8\% IC 55.3-71.6. Dicha intervención es característica en el hospital de agudos descripta y fundamentada por la autora del trabajo de investigación dado que han sido ampliamente investigados los efectos de la hospitalización (Pérez, 2006) y a través de la educación, entre otras intervenciones, se pretende prevenir, disminuir las complicaciones por el reposo.

A pesar de ello, hay algunas razones que condicionan que se pueda realizar el asesoramiento arriba explicitado, a saber: deterioro cognitivo, variación del estado de conciencia (confusión a coma) del paciente; y/o ausencia de cuidador.

Cabe mencionar que no se refleja en esta investigación la frecuencia en que se llevan a cabo las interven- 
ciones en educación en equipamiento de soporte vital y uso correcto de la mecánica corporal, las que se realizan más en la práctica clínica diaria según la prevalencia que se presenta [16.3\% IC 10.8-23.7 y $9.2 \%$ IC $5.2-15.6]$ respectivamente. En este caso, dichos resultados podrían justificarse como un sub registro en la documentación escrita (con esto me refiero a que se pueden llevar a cabo pero que no se documenta). Estos resultados sorprendieron a la investigadora, dado que no se corresponde con lo que se observa en la asistencia diaria.

\section{Intervención Métodos Preparatorios}

Las intervenciones incluidas en los métodos preparatorios que se realizan con mayor frecuencia son el posicionamiento: $51,8 \%$ IC 43.2-60.2, y esto coincidentemente con lo que describen las TO canadienses (Giesbrecht, B., 2006), se sustenta en evitar el desarroIlo de las úlceras por presión por una parte, y por la otra contribuir a la disminución de los efectos del reposo en cama: como por ejemplo, las infecciones respiratorias, se sabe que la capacidad funcional residual disminuye en decúbito supino hasta un $30 \%$ en comparación con la posición de decúbito lateral en la que la disminución es sólo del $17 \%$. Esto resalta la importancia de los cambios posturales frecuentes en la prevención y tratamiento de las complicaciones respiratorias (Pérez, 2006).

Obviamente siendo que hay pacientes con posibilidad de involucrarse en actividades de movilidad funcional (darse vuelta en la cama, sedestar, caminar) no se lleva a cabo esta última intervención en el 100\% de los casos y a pesar de ello el porcentaje en la muestra y proyección a la población es elevado.

Si bien la intervención de Adaptaciones ambientales: cambios en el mobiliario, alcance de elementos, apoya pie a fin de promover la participación en actividades significativas se registra en un $48,6 \%$, IC 39.8 56.8, considero que, como ocurre en la estimulación del alerta, orientación témporo-espacial existe un subregistro dado por la naturalidad en que se realiza la intervención, donde existe una subvaloración por parte de las terapeutas en considerarla para la documentación. En varias ocasiones para promover las actividades realizamos cambios en el entorno físico, que, a la hora de documentar se releva la actividad que se pudo llevar a cabo y no cómo se modificaron las demandas de la misma para lograrlo. Quizá estos resultados contribuyan a concientizar la importancia de registrar más detalladamente todos los aspectos donde intervenimos a fin de poder en un futuro describir más precisamente la intervención de TO.

Por otra parte también se relaciona con el tipo de patología, hay algunas de ellas, como las traumatológicas que tienen más prevalencia de estas intervenciones como la indicación de férulas, adaptaciones. En este estudio la mayoría de la población era de las salas de clínica médica y no de traumatología.

\section{Uso terapéutico del YO}

Al observar los resultados de las intervenciones que abarca el uso terapéutico del yo observamos que la escucha activa es una de las intervenciones que se llevan a cabo con mayor frecuencia. Consideramos que esta destreza comunicacional es vital en la práctica hospitalaria y no es específica de una profesión. Pareciera que en ocasiones se considera que el psicólogo es el profesional encargado de esta intervención. Particularmente si se comprende el alcance de la misma se entenderá que todo profesional debe tener competencia en esta intervención. En lo que respecta a terapia ocupacional el paciente encuentra en la sesión y según lo observado en mis años de experiencia clínica, un espacio donde explicita sus sensaciones, sentimientos, temores en relación con el grado de participación en sus actividades, la satisfacción en las mismas, las creencias futuras en relación a su desempeño. Es cierto que todo esto genera diversas emociones tales como ansiedad, angustia, depresión, negación y es inevitable gatillarlas en relación a su nivel de dependencia/participación. (Beneit Medina, P., Latorre Postigo, JM., 1994; Do Prado De Carlo y otros, 2004; Latorre Postigo, JM., 1994). Es por ello que, dar apoyo, soporte a través de la escucha es lo básico para, posteriormente, vehiculizar la necesidad de un profesional calificado para abordarlas como lo son los profesionales del área de Salud mental. En nuestra muestra se registró un $46,8 \%$ de escucha activa, IC 38.4-54.4\%, es importante señalar y esto a los fines del modo de registro de datos, que impresiona que hay un subregistro de estas intervenciones dado que en la formación del TO (en las Universidades de Argentina) no se identifican como incumbencia profesional per se y sí se desarrollan en el pos grado. Es por ello que documentarlas lleva más tiempo que el que lleva implementarlas.

Otra intervención que se destaca dentro de las de uso terapéutico del yo se centra en la vehiculización de ne- 
cesidades. Esta intervención constituye un ejemplo claro relacionado con el tipo de población que asistimos cotidianamente y que también se refleja en la muestra estudiada. Las particularidades de los hospitales de agudos (Latorre Postigo, JM., 1994) con la disponibilidad de recursos físicos, humanos, de mobiliario, de escasa articulación entre los profesionales tratantes, entre otros, T.O funciona como nexo para proporcionar cierta mejora en la estadía hospitalaria proporcionando información a las distintas partes involucradas, sugiriendo estrategias para gestión de elementos, entre otros.

\section{Intervención de consultoría}

Es cierto que en esta investigación las intervenciones relacionadas a consultoría tienen muy baja frecuencia; quizá algunas razones de esta situación puedan deberse a una mala interpretación de términos por parte de las profesionales TO y falta de entrenamiento en el uso de la terminología, donde se toma como sinónimos educación y consultoría y, por lo tanto, se documenten intervenciones de consultoría como educación.

Esto por una parte, y por la otra, es cierto que el grado de participación para implicarse en un tratamiento, para sentirse protagonista en encontrar alternativas en la resolución de problemas en relación a su salud, es difícil de encontrar en la mayoría de la población que asiste al hospital público, altamente vulnerable no sólo por la situación de enfermedad, que los hace más pasivos y dependientes del supuesto saber de los profesionales, sino también por las características culturales, nivel educativo, edad, modelos de asistencia paternalistas y asimétricos (Beneit Medina, 1994) predominantes en las instituciones de salud de nuestro país que podrían también estar interviniendo.

Por otro lado, también es real que, de acuerdo al modelo de salud vigente, el modelo paternalista, es más sencillo dar directivas de cómo cuidarse que, en conjunto, construir con el saber del paciente su propio cuidado.

También se sabe hoy en día, que este sistema, donde el lugar del saber está ubicado en los profesionales, y en los pacientes un mero rol pasivo, no ha dado los mejores resultados en relación a adherencia de tratamiento; es por ello que cada día se intenta desarrollar más estrategias para implicar a los pacientes en sus tratamientos. En relación a este punto sería importante ahondar en la investigación.

\section{Relación de edad e Intervenciones frecuentes}

De lo cotejado entre este estudio y nuestra práctica clínica diaria en el hospital, coinciden en gran parte los resultados que relacionan la edad y las intervenciones usadas con mayor frecuencia. Así mismo avalan la necesidad de considerar estos resultados dado que es importante considerar que a mayor edad, mayor riesgo a desarrollar complicaciones como se presentan en los siguientes trabajos (Affleck \& cols, 1986; Byon, S., Wilding, C. \& Eyres, L., 2007; Gillis, A., MacDonald, B. \& Macksaac, 2005; Gillis \& cols, 2008; Lim, SM., Doshi, V., Castasus, B. \& Lim JKH., 2006).

De alguna manera que las intervenciones que se realicen con mayor frecuencia estén destinadas a adultos mayores nos estaría hablando que TO intenta cierta prevención, obviamente dicha efectividad deberá ser probada en otros trabajos, aquí sólo se refleja que se realizan.

La utilización de las AVD en sala con adultos mayores se fundamenta en pretender involucrarlos para que continúen siendo activos y así prevenir otras complicaciones, en nuestro trabajo se muestra que a mayor edad más uso de AVD, además los más jóvenes por lo general son más independientes o tienen más iniciativa para involucrarse.

La misma explicación puede aplicarse a la indicación de uso de adaptaciones ambientales y personales.

El método preparatorio posicionamiento también resultó presentar diferencias en relación a los promedios de edades usándose en los adultos mayores con más frecuencia. Esto se explica por un lado porque ellos son más dependientes, o sus síntomas les impiden moverse, su iniciativa, su temor, entre otras. También sabemos que nosotros implementamos esta intervención, porque con el simple cambio de posición prevenimos úlceras por presión, contracturas, deformidades, e infecciones respiratorias, colaboramos a realizar AVD seguras: ejemplo, cuando se alimentan y los reposicionamos porque están descendidos previniendo el riesgo de aspiración.

En lo que respecta a la estimulación de la alerta (que incluye la orientación témporo-espacial, el despertar durante el día) se observa que si bien los grupos son muy dispares $\left(\mathrm{n}^{\circ} 18\right.$ recibió la intervención vs $\mathrm{n}^{\circ} 123$ no la recibió) se encuentran diferencias significativas. Creo que hay en este punto un subregistro de intervención en el grupo de adultos mayores porque es una práctica que se realiza cotidianamente quizá naturalizándose y subregistrando en la documentación. 
Siguiendo con las diversas investigaciones sobre el desacondicionamiento y la población adulta mayor, quien es uno de los grupos identificados como de alta vulnerabilidad (Gillis \& cols, 2008; Lim \& cols, 2006; Pérez, 2006; Sager, M., Rudberg, MA., Jalaluddin, M., Franke, T., Inouye, S. Landefeld, CS., Siebens, H. \& Winograd, C., 1996), más expuestos a riesgos, nuestros resultados muestran intervenciones para minimizar este riesgo, nuevamente que esto sea exitoso o no será el foco de próximas investigaciones, sí sabemos que estamos haciendo intervenciones con mayor frecuencia en esta población.

\section{Relación entre presencia de cuidador e intervenciones prevalentes}

Analizando los resultados del objetivo que investiga la presencia de cuidador y la frecuencia de intervenciones, si bien las dos presentan diferencias significativas en cuanto a la presencia, son muy descriptivas de la instrumentación de intervenciones en nuestros hospitales de la CABA, Argentina.

Sin embargo, cabe mencionar que hay algunas intervenciones que se llevan a cabo y no han presentado diferencias en la investigación, como ser educación en la mecánica corporal y uso de equipamiento de soporte vital, que como expresaba más arriba impresiona que se debe a un sub registro en la documentación, porque tampoco está representada en la frecuencia de la intervención.

En el caso de educación en uso correcto de la mecánica corporal se realizó en 13 oportunidades y 12 de ellos tenían cuidador. El problema es que había grandes diferencias en los grupos, lo que podría tratarse de un error beta, es por ellos que podría suponerse lo mismo con respecto a uso de equipamiento de soporte vital: de 23 veces que se registró que se llevaba a cabo la intervención, en 19 casos tenían cuidador, el problema es que el grupo que no recibió la intervención es superior: 118 personas.

\section{Agradecimientos:}

Dra. Nora I. Castiglia por el asesoramiento metodológico.

\section{ReferenCias BibliográficAs}

Affleck A.; Lieberman, JP.; Rohrkerper, K. (1986). Providing Occupational Therapy in an Intensive Care Unit. The American Journal of occupational Therapy, 40(5), 323-332.

Beneit Medina, P. (1994). Conductas de Salud, en Latorre Postigo, J.M.; Beneit Medina, P.J. (Ed). Psicología de la Salud (27-36). Buenos Aires. Argentina: Lumen.

Beneit Medina, P. (1994). La Enfermedad Crónica, en Latorre Postigo, J.M.; Beneit Medina, P.J. (Ed). Psicología de la Salud (225-237). Buenos Aires. Argentina: Lumen.

Beneit Medina, P.; Latorre Postigo (1994). El enfermo terminal y muerte, en Latorre Postigo, J.M.; Beneit Medina, P.J. (Ed). Psicología de la Salud (319-333). Buenos Aires. Argentina: Lumen.

Bondac, S.; Hermann, V.; Frost, L.; Lashgari, D.; Finnen, L.; Alexander, H. (2009). Occupational Therapy in Acute Care USA: American Occupational therapy Association.

Byon,S.; Wilding,C.; Eyres, L. (2007). An innovative occupationfocussed service to minimize deconditioning in hospital: challenges and solutions. Australian Occupational therapy Journal, 54, 225-227.

Do Prado De Carlo,M.; Camargo Bartalotti, C.; Muñoz Palm, RdC A (2004). Terapia Ocupacional em Reabilitacao Física e Contextos Hospitalares: Fundamentos para la Práctica. (5-28), en Do Prado de Carlo, M.; Miranda Luzo, M.C. Terapia Ocupacional Rehabilitacao Física e Contextos Hospitalares. Sao Paulo Brasil: Roca Ltda.

Eyres, L.; Unsworth, C. (2005). Occupational therapy in acute hospitals: The effectiveness of a pilot program to maintain occupational performance in older clients. Australian Occupational Therapy Journal 52, 218-224.

Fasoli, S. (2008). Restoring Competence for Homemaker and Parent Roles en Radomsky MV; Lathamn Trombly C.A. Occupational Therapy for Physical Dysfunction. (855-874) USA: Lippincot. Williams \&Wilkins.

Fernández C. M.; Fernández Huete, J. (2005). Marco de Trabajo para la Práctica de la Terapia Ocupacional: Ámbito de Competencia y Proceso. www.Terapia-Ocupacioanl.com

Flimn, N. Jackson, J.; Gray, J.; Zemke, R. (2008). Optimizing Abilities and Capacities: Range of motion, Strength and Endurance en Radomsky M.V.; Lathamn Trombly C.A. Occupational Therapy for Physical Dysfunction. USA: Lippincot. Williams \&Wilkins (573-597).

Gage, M.; Cook Valiant, J.; Friday-Field, K.; (1997). Understanding the transition to Community Living After Discharge From an Acute Care Hospital: An Exploratory Study. Am J Occup Therapy, 51 (2), $96-103$. 
Giesbrecht (2006). Pressure ulcers and occupational therapy practice: A Canadian perspective. Canadian Journal of Occupational Therapy 73 (1), 56-63.

Gill., T; Allore, H.; Guo, Z. (2004) The deleterious effects of bed rest among community-Living Older Persons. Journal of Gerontology Medical Sciences. 59 A (7), 755-761.

Gillis A.; MacDonald B.; Macksaac (2005) Prevention Deconditioning in the Hospitalizaded Elderly. Can Nurse, 101(6),16-20.

Gillis A.; MacDonald B.; Macksaac (2008). A Nurses'Knowledge, Attitudes, and Confidence regarding Preventing and treating Deconditiong in older Adults. The Journal of Continuing Education in Nursing,39 (12), 547-554.

Griffin,S. (1993). Short Bed Stays: Their Effects on Occupational Therapy Services in Teaching Hospitals. Arch Phys Med Rehabil, 74 1087-90.

Griffin, S.; (2002). Occupational Therapy practice in Acute Care neurology and Orthopaedics Journal of Allied Health, 31 (1), 35-42.

Griffin, S Mcconnell, D. (2001). Australian occupational therapy practice in acute care settings. Occupational Therapy International, 8 (3), 184-197.

James, A.B. (2008) Restoring the Role of Independent Person en Radomsky M.V.; Lathamn Trombly CA Occupational Therapy for Physical Dysfunction. USA: Lippincot. Williams \&Wilkins (774-816).

Kortebein, P. (2009). Rehabilitation for Hospital-Associated Deconditioning. Am J Phys Med Rehabil, 88 (1), 66. 77.

Lanier P. S. (2008). Restoring Mobility en Radomsky M.V.; Lathamn Trombly C.A. Occupational Therapy for Physical Dysfunction. USA: Lippincot. Williams \&Wilkins (817-853).

Latorre P., J.M. (1994). El Paciente en el medio hospitalario, en Latorre Postigo, J.M.; Beneit Medina, P.J. (Ed). Psicología de la Salud (205-214). Buenos Aires. Argentina: Lumen.

Lim S.M.; Doshi V.; Castasus B. Lim JKH (2006). Factors causing delay in Discharge of Elderly patients in an Acute Care Hospital. Ann Acad Med, 35 (1), 27-32.

López R., D. (2000). Técnicas de respiración. Estrés. Epidemia del Siglo XXI. Buenos Aires, Argentina: LUMEN.

López R. D. (2000). Técnicas relajación neuromuscular. Estrés. Epidemia del Siglo XXI. Buenos Aires, Argentina: LUMEN.

López R. D. (2000). Técnicas meditación y visualización. Estrés. Epidemia del Siglo XXI. Buenos Aires, Argentina: LUMEN.

Lynn, Y. (2008). Rheumatoid Arthritis, Osteoarthritis, en Radomsky M.V.; Lathamn Trombly C.A. Occupational Therapy for Physical Dysfunction (1214-1243). USA: Lippincot. Williams \&Wilkins.

Maher,C.; Bear-Lehman, J. (2008). Orthopaedic Conditions en Radomsky M.V.; Lathamn Trombly C.A. Occupational Therapy for Physical Dysfunction (1123). USA: Lippincot. Williams \&Wilkins.
Ocello, M.; Rolfi,F. (2006). Terapia Ocupacional en un Hospital de pacientes agudos. Revista Gallega de Terapia Ocupacional. (4) 1-8.

Pérez, M. (2006). Terapia Ocupacional en un Hospital General de Agudo. Manuscrito no publicado. Buenos Aires. 1-39.

Pérez, M.; Casal, C.; Cirone, M.; González, A.; Parranquini, L.; Bracco, F.; Vadell, A.; Oromi, M.; (2008). Instructivo ficha Evaluación Clínica Médica. Terapia Ocupacional Hospital General de Agudos Bernardino Rivadavia. Manuscrito no publicado. Buenos Aires, Argentina.

Piedrola, G. (2001). Medicina Preventiva y Salud Pública. Ed. Masson-Salvat. España.

Quintana, L.A. (2008). Optimizing Vision, Visual Perceptual and Praxis Abilities, en Radomsk y, M.V.; Lathamn, Trombly C.A. Occupational Therapy for Physical Dysfunction USA: Lippincot. Williams \& Wilkins (728-747).

Radomski, M.V.; Schold Davis, E. (2008). Optimizing Cognitive Abilities en Radomsky MV; Lathamn Trombly C.A. Occupational Therapy for Physical Dysfunction. USA: Lippincot. Williams \& Wilkins (748-773).

Rausch, G. Melvin J. (1986). A new era in acute Care The American Journal of Occupational Therapy, 40 (5), 319-322.

Sager, M. A.; Rudberg, M. A.; Jalaluddin, M.; Franke, T.; Inouye, S. K.; Landefeld, C. S.; Siebens, H.; Winograd, C. (1996). Hospital Admission Risk Profile (HARP): Identifying Older Patients at Risk for Functional Decline Following Acute Medical Illness and Hospitalization J Am Geriatric Soc, 44 (3), 251-257.

Sutton, A. (1998). An acute medical Admission Unit: is there Place for an Occupational Therapy? British Journal of Occupational Therapy, 61 (1), 2-6. 